\title{
204 - Social Health and Dementia
}

\author{
Mapping the complexity of factors influencing cognitive functioning in dementia with a special focus \\ on social health \\ Karin Wolf-Ostermann, Prof. Dr., wolf-ostermann@uni-bremen.de, Health Sciences Bremen, University \\ of Bremen, Germany.
}

Henrik Wiegelmann, M.A., Health Sciences Bremen, University of Bremen, Germany, hwiegelmann@uni$\underline{\text { bremen.de }}$

Imke Seifert, M.A, Health Sciences Bremen, University of Bremen, Germany, imke.seifert@unibremen.de

Dorota Szczesniak, PhD, Department of Psychiatry, Wroclaw Medical University, Poland, dorota.szczesniak@umed.wroc.pl

Marta Lenart, Department of Psychiatry, Wroclaw Medical University, Poland, marta.lenart@umed.wroc.pl

Mateusz Luc, Department of Psychiatry, Wroclaw Medical University, Poland, mateusz.luc@student.umed.wroc.pl

Marcin Pawlowski, Department of Psychiatry, Wroclaw Medical University, Poland, marcin.pawlowski@student.umed.wroc.pl

Etienne Rouwette, Prof. Dr., Radboud University, Nijmegen, The Netherlands, e.rouwette@fm.ru.nl

Ansgar Gerhardus, Prof. Dr., Health Sciences Bremen, University of Bremen, Germany, ansgar.gerhardus@uni-bremen.de

Joanna Rymaszewska, Prof. Dr., Department of Psychiatry, Wroclaw Medical University, Poland, joanna.rymaszewska@umed.wroc.pl

and on behalf of the SHARED Consortium

Introduction: Over the past years the scientific discourse on health moved beyond unidimensional approaches, focusing on a more complex bio-psycho-social understanding. Regarding dementia and cognitive health, several studies have shown that various multidimensional factors (risk/protective) and their interactions, as well as existing individual and social resources, contribute to the heterogeneity observed for onset and further course of dementia. This presentation will a) give a systematic overview of causal factors in relation to the onset and progression of cognitive functioning and dementia b) show results of a first generic system dynamics model as Causal Loop Diagram (CLD) visualizing how different factors of the system are interrelated.

Methods: A mixed methods approach was used. First, we conducted a systematic literature review on factors influencing cognitive health with an emphasis on social factors. Second, the Group Model Building (GMB) approach was used to further elaborate the knowledge base from the literature review and to start building a Causal Loop Diagram as a first comprehensive system dynamics working model. In a final third phase we integrated both strands, agreed on the knowledge base by stepwise discursive consensus and created the CLD.

Results: The CLD model includes 73 unique factors directly or indirectly influencing cognitive functioning. Those factors show the basic causal structure of the interplay of variables present in the 
development and trajectory of dementia. These factors could be grouped into six thematic/disciplinary clusters, such as personal factors, socioeconomic factors, lifestyle factors, neuro-bio-medical factors, environmental factors and social health factors. The model indicates a significant role of social health indicators for cognitive health in dementia.

Conclusion: The generic CLD model reflects the knowledge of a multidisciplinary group of researchers, merged with results from a systematic literature overview and supplemented by discussion and iterative feedback processes. The mixed methods approach as well as the integration of system thinking methods turned out to be a reasonable approach to develop and graphically represent the complex structure of factors influencing cognitive functioning in dementia. This model facilitates the development of novel hypotheses about causal relationships between social health and dementia.

Translational dementia research on social health and cognitive functioning in rodents and humans Myrra Vernooij-Dassen ${ }^{1}$, Suzanne Lanooij ${ }^{2}$, Isabelle van der Velpen ${ }^{4}$, Rene Melis ${ }^{1}$, Pim Drinkenburg ${ }^{2,3}$, Uli Eisel $^{2}$, Arfan $\mathrm{Ikram}^{4}$, Marieke Perry ${ }^{1}$, Eddy van der Zee ${ }^{2}$, Martien Kas ${ }^{2}$

1 Radboud Univerity Medical Center Nijmegen, the Netherlands

${ }^{2}$ Groningen Institute for Evolutionary Life Sciences, University of Groningen, the Netherlands

${ }^{3}$ Janssen Research \& Development, a Division of Janssen Pharmaceutica NV, Beerse, Belgium

${ }^{4}$ Erasmus University, Department of Epidemiology, the Netherlands

\section{Rational}

The paucity in dementia research requires finding new knowledge on factors that influence the onset of dementia and on related interventions. One of these factors might be social health. New neurobiological insights in this area can be derived from studying social health in rodents. To determine the crossspecies translational value of social health, in this project, homologies between social health in rodents and humans are being investigated.

\section{Aim}

The aim of the study presented in this symposium is to identify social behavioural components in rodents in order to eventually derive and test hypotheses on the relationship between social health, brain mechanisms, cognitive functioning and dementia in the overall study.

\section{Methods}

A first step is the adaptation of the definition of social health in order to allow use of this concept in rodent research. A next step is the selection of observational markers reflecting social health.

\section{Results}

Departing from a definition of social health as domain of positive health (Huber) we adapted the concept of social health by focussing on its actors: the individual and the social environment. Social health relates to the influence the competencies of the individual to participate in social activities and the influence of interactions with the social environment have on the dynamic balance between capacities and limitations. Quantifiable features of social behaviour in rodents concern hierarchy, social approach and avoidance behaviour, aggressive behaviour, sexual behaviour, among others. Interestingly, the core neural circuitries in the brain regulating (healthy) social behaviours have been identified, and seem evolutionary well-preserved, thus showing substantial neuro-anatomical overlap between distant species.

\section{Conclusion}

Comparison between rodent and human behaviour reveals comparable behaviour of rodents and humans such as social companionship, information on the emphasis on the physical expression of 